\title{
Twenty four hour blood pressure monitoring in normal tension glaucoma
}

\author{
Jörg Heinrich Meyer, Johannes Brandi-Dohrn, Jens Funk
}

\begin{abstract}
Background-The few investigations that used continuous 24 hour blood pressure monitoring to investigate whether blood pressure in patients with normal tension glaucoma is lower than in normal subjects yielded conflicting results. Therefore, a prospective controlled trial was carried out.

Methods-Systemic blood pressure was recorded continuously over a 24 hour period in 20 patients with normal tension glaucoma (IOP $\leq 21 \mathrm{~mm} \mathrm{Hg}$ ). Eight of them showed a localised loss of the neuroretinal rim area and, in addition, optic disc haemorrhages-that is, focal ischaemic signs. Twenty healthy patients without glaucoma, who were hospitalised for cataract or retinal surgery, served as controls. Blood pressure was automatically measured every 20 minutes during the day and every $\mathbf{4 0}$ minutes at night.

Results-Both groups showed a significant (physiological) blood pressure drop at night, which was significantly $(p<0.001$, ANOVA) more pronounced in the group with normal tension glaucoma than in the control group. There was a weak trend towards lower blood pressure values in the normal tension glaucoma group. Minima, maxima, and mean values of the systolic, diastolic, and mean arterial pressures did not differ significantly between the group with normal tension glaucoma and the control group. The greatest differences occurred with nocturnal systolic and diurnal diastolic values. There were no significant differences between the subgroup with focal lesions and the other patients with normal tension glaucoma.

Conclusions-Patients with normal tension glaucoma tend to have lower blood pressure values ( $>0.05$, ANOVA) than normals; this difference is probably much smaller than formerly assumed. Patients with normal tension glaucoma, however, have significantly greater nocturnal blood pressure drops $(p<0.001$, ANOVA) than normal controls. Nocturnal blood pressure drops (relative day-night differences)
\end{abstract}

Accepted for publication 24 May 1996 may play a more important role in the pathogenesis of normal tension glaucoma than the absolute height of the blood pressure.

(Br $\mathcal{F}$ Ophthalmol 1996;80:864-867)

Today we believe that glaucomatous damage occurs not only as the result of increased intraocular pressure but also because of other, mainly vascular, factors. These include arteriosclerosis, ${ }^{1}$ coagulative disorders, ${ }^{2}{ }^{3}$ or other general diseases ${ }^{4}{ }^{5}$ and vasospasms. ${ }^{6}{ }^{7}$ Some previous studies demonstrated lower systemic blood pressure values in normal tension glaucoma (NTG) than in normals or high tension glaucoma. ${ }^{8-10}$ There are, however, only few investigations using continuous 24 hour blood pressure monitoring. ${ }^{11-14}$ Until now in only one study $^{13}$ was a normal tension glaucoma group compared with a matched control group. In this study significantly lower blood pressure readings were found in the NTG group. This contradicts another study, which showed blood pressure values of NTG patients to be well within the ranges reported for control subjects in the (cardiological) literature. ${ }^{15}$

As this subject is relevant for the understanding and management of normal tension glaucoma we decided to set up a new prospective, strictly controlled study and analysed arterial blood pressure in all patients with normal tension glaucoma being hospitalised in our clinic in 1994 and in a matched control group.

\section{Patients and methods}

We continuously recorded systemic blood pressure in 22 consecutive patients who were hospitalised at the university eye clinic Freiburg in 1994 because of normal tension glaucoma. Demographic data are summarised in Table 1. All patients (aged 67 (SD 11) years, 13 female, seven male) had unilateral or bilateral glaucoma (typical excavation of the optic disc and/or glaucomatous visual field defects) and intraocular pressure (IOP) $\leq 21$ $\mathrm{mm} \mathrm{Hg}$ within a 48 hour profile, comprising at least five values including one at midnight. Other causes of optic atrophy were excluded on the basis of (normal) computed tomography scans and history (high IOP in the past, trauma, intoxication, ischaemia).

The reason for admission was progressive visual field damage despite normal IOP. Progression was defined clinically by an experienced ophthalmologist (JHM) based on a significant decrease in neuroretinal rim area, measured by the Rodenstock optic nerve head analyser (ONHA, linear regression analysis) and/or a visual field deterioration. At least 


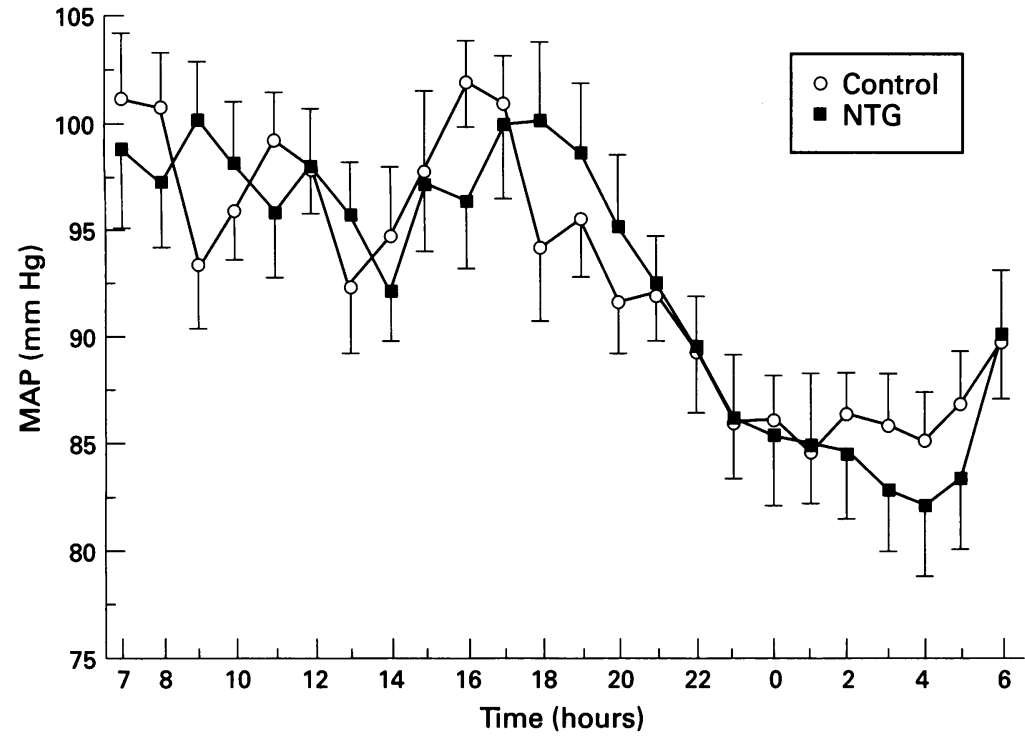

Figure 1 Time course of mean arterial pressure (MAP) in patients with normal tension glaucoma (NTG) and normals (SEM).

three Octopus (G1X program) or Goldmann visual fields had to be available, a deterioration (increase of the mean defect $\geq 1 \mathrm{~dB}$ and/or increase of a local defect-that is, a sensitivity loss $\geq 3 \mathrm{~dB}$ at three or more adjacent test points in the upper or lower hemifield) had to be present in two consecutive visual fields.

Two patients were excluded. Exclusion criteria were diabetes mellitus and severe general diseases that might influence blood pressure. To avoid a bias towards lower blood pressure values treatment against arterial hypertension (or hypotension) was not a criterion for exclusion.

Eight of the remaining 20 patients showed a localised loss of neuroretinal rim area ('notch') and seven of these eight patients had one or more optic disc haemorrhages. According to Spaeth $^{16}$ we called this subtype of normal tension glaucoma 'focal ischaemic glaucoma' (FIG), knowing that this entity is believed to occur not only in NTG patients but also in some others with moderately elevated IOP. ${ }^{17}$

Twenty generally healthy patients (aged 67 (SD 11) years, 14 female and six male) without glaucoma, who were hospitalised for cataract or retinal surgery, served as controls. There was no difference to the NTG group with respect to age, sex, race (all were white), surroundings (all were hospitalised in the same clinic at similar times of year and the exclusion criteria mentioned above. Like in the NTG group eight of the 20 patients were taking a mild antihypertensive therapy (Table 1 ). The drugs and their dosage were similar (six times monotherapy mainly with calcium channel blockers or $\beta$ blockers, two times a combination of two drugs) in both groups. Medication was taken in the morning or at several times of day but never in the evening alone.

The interval between surgery and blood pressure recording was at least 2 days to avoid any influences of the operation (medication, mental stress).

Blood pressure and heart rate were measured at the left brachial artery with a portable
Table 2 Comparison (paired t test) of the 'relative difference' ((day - night)/(day + night)) between patients with normal tension glaucoma (NTG) and normals

\begin{tabular}{llll}
\hline & NTG group & Control & $p$ Values (t test) \\
\hline Systolic: & & & \\
$\quad$ Mean & 0.051 & 0.034 & 0.19 \\
Max & 0.097 & 0.052 & 0.016 \\
$\quad$ Min & 0.017 & 0.01 & 0.71 \\
Diastolic: & & & \\
$\quad$ Mean & 0.094 & 0.055 & 0.03 \\
Max & 0.186 & 0.079 & 0.001 \\
Min & 0.058 & 0.027 & 0.14 \\
MAP: & & & \\
Mean & 0.07 & 0.053 & 0.31 \\
Max & 0.133 & 0.052 & 0.002 \\
$\quad$ Min & 0.041 & 0.023 & 0.31 \\
Heart rate: & 0.083 & 0.073 & 0.46 \\
$\quad$ Mean & 0.03 & & \\
\hline
\end{tabular}

automatic device (90207-32 monitor, Spacelabs Inc) every 20 minutes during the day (6 am to $10 \mathrm{pm}$ ) and every 40 minutes at night (10 pm to $6 \mathrm{am})$.

The following factors were used for further evaluation:

1 The highest, lowest, and mean values were calculated separately for the systolic, diastolic, and mean arterial pressures of each patient ( $\mathrm{MAP}=$ diastolic pressure $+1 / 3$ (systolic pressure/diastolic pressure)), respectively.

2 All the above were calculated separately for daytime and night-time.

3 Means and standard errors were determined for all factors in the normal group and in the NTG group (all, non-FIG, and FIG subgroups, respectively).

Statistical comparison of all discrete variables was made with the unpaired Student's $t$ test and ANOVA for repeated measurements. The paired $t$ test was used only when the diurnal values of each patient were compared with the nocturnal ones.

\section{Results}

In both the NTG and the control group we found a physiological blood pressure drop at night (Fig 1), which was highly significant ( $p<0.01$, paired $t$ test) for all factors except for the minimal systolic pressure in NTG patients and in controls. The nocturnal blood pressure drop was more pronounced in the normal tension glaucoma group $(11 \mathrm{~mm} \mathrm{Hg}$ systolic/13 $\mathrm{mm} \mathrm{Hg}$ diastolic) than in controls $(8 \mathrm{~mm} \mathrm{Hg}$ systolic and diastolic).

To compare the nocturnal blood pressure drops between both groups we calculated a 'relative difference' of the blood pressure values ((day - night)/(day + night)). These relative differences were significantly larger in the NTG group than in the control group (ANOVA, $p=0.0002$ ). This significant difference between both groups remained when the patients on blood pressure treatment were excluded (ANOVA, $\mathrm{p}=0.0026$ ).

For the mean and maximal diastolic blood pressures, the maximal systolic blood pressures, and the maximal MAP the relative difference between both groups was significant in the unpaired $t$ test (Table 2, Fig 2).

The relative differences tended to be greater in the FIG subgroup than in the other NTG patients, though not significantly (ANOVA, $\mathrm{p}=0.15)$. 


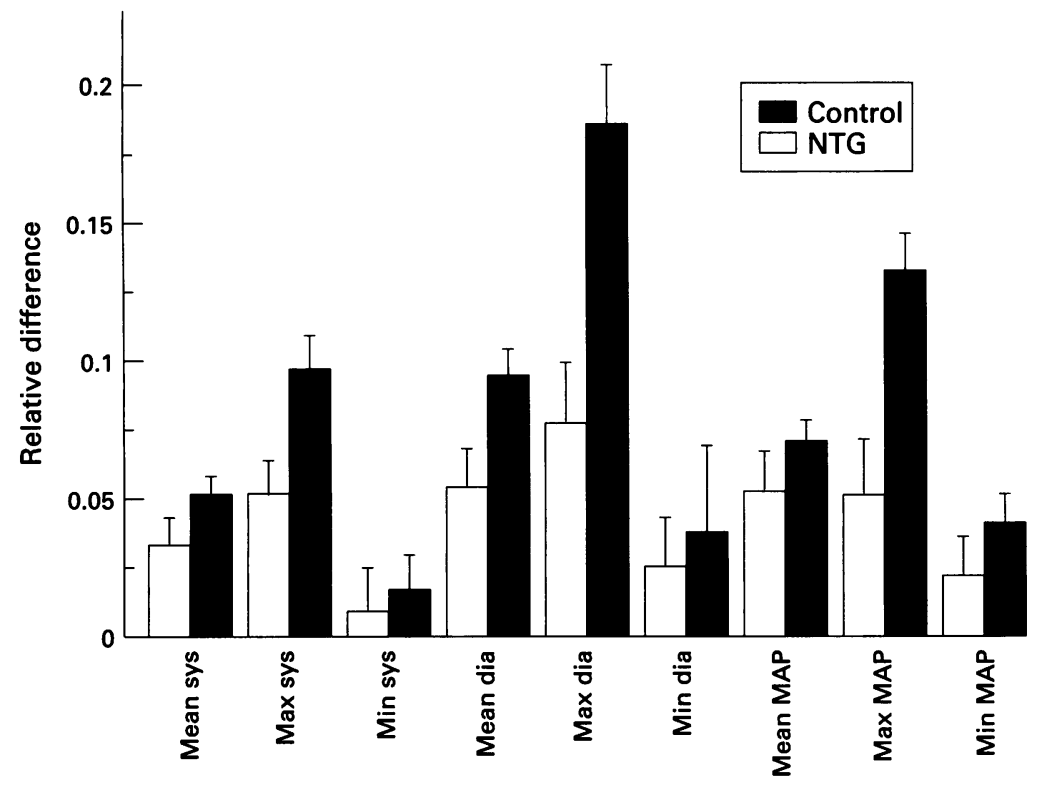

Figure 2 'Relative differences' ((day - night)/(day + night $))$ of the systolic, diastolic, and mean arterial pressure (MAP) in normal tension glaucoma (NTG) and control patients. Calculation is made for the maxima, minima, and mean values of each factors (SEM).

When comparing the absolute values there was a weak (not significant) general trend towards lower blood pressure values in the NTG group. The greatest differences occurred with nocturnal systolic values (Table 3 ). For all variables, however, the differences between the NTG group and the control group were not significant ( $p=0.94$, ANOVA). An exception was the maximal diastolic blood pressure during the day which, against the general trend, was higher in the NTG group.

There were no significant differences $(p=0.5$, ANOVA) between the subgroup with focal ischaemic signs (FIG) and the other patients with normal tension glaucoma (nonFIG patients, Table 3).

Heart rate declined at night but did not differ significantly between NTG patients and normals, or between FIG and non-FIG (Table 3).

\section{Discussion}

As with normal people reported in the cardiological literature ${ }^{15}$ our patients and controls showed the physiological nocturnal drop in blood pressure (dip), which is presumably caused by a reduced sympathetic activity during sleep. ${ }^{18}$ This drop was significant for all factors except for the minimal systolic pressure in NTG patients and controls.

Nocturnal drops ('relative differences') were significantly higher in the NTG group confirming a report (on four patients) by Kaiser and Flammer, ${ }^{19}$ who found nocturnal blood pressure drops to be the only risk factor in patients with rapidly developing optic nerve damage. Graham et al recently reported significantly greater nocturnal blood pressure drops in glaucoma patients with progressive visual fields than in glaucoma patients with stable visual fields. ${ }^{14}$ They did not compare NTG patients with a matched control group, however.

Interestingly, the nocturnal decrease of diastolic blood pressure has previously been reported to be significantly greater in NTG patients than in patients with anterior ischaemic optic neuropathy. ${ }^{12}$

It should be mentioned that the hypotensive treatment may affect blood pressure and our results. Hypotensive treatment may produce nocturnal dips. It may, however, also lower blood pressure during the day. According to a study of Tochikubo et $a l^{20} \beta$ blockers had a night-time/daytime blood pressure reduction effect of $0.65-0.74$, calcium antagonists had night-time/daytime ratios of $0.89-1.2$, and ACE inhibitors 0.81-0.97. This might imply that, for example, $\beta$ blockers are less likely to produce nocturnal hypotension than calcium antagonists.

We did not exclude patients with (of course usually treated) essential arterial hypertension from the study, because the result would be interesting if hypertensive patients (on treatment) were present significantly more often in

Table 3 Mean values of all blood pressure variables (SEM). p Values of an unpaired comparison ( $t$ test) between all NTG patients and normals and between FIG and non-FIG patients within the NTG group

\begin{tabular}{|c|c|c|c|c|c|c|}
\hline & \multicolumn{3}{|l|}{$N T G$} & \multirow{2}{*}{$\begin{array}{l}\text { Normals } \\
(n=20)\end{array}$} & \multirow{2}{*}{$\begin{array}{l}p \text { Value } \\
\text { All v normal }\end{array}$} & \multirow{2}{*}{$\begin{array}{l}\text { p Value } \\
\text { FIG v non-FIG }\end{array}$} \\
\hline & $A l l(n=20)$ & $F I G(n=8)$ & non-FIG $(n=12)$ & & & \\
\hline \multicolumn{7}{|l|}{ Systolic: } \\
\hline \multicolumn{7}{|l|}{ Day } \\
\hline mean & $129(3.0)$ & $133(6.0)$ & $128(2.9)$ & $131(2.7)$ & 0.69 & 0.40 \\
\hline $\max$ & $163(5.4)$ & $170(12.4)$ & $158(3.5)$ & $160(4.8)$ & 0.65 & 0.29 \\
\hline $\min$ & $105(2.2)$ & $105(4.4)$ & $105(2.5)$ & $108(3.4)$ & 0.43 & 0.99 \\
\hline \multicolumn{7}{|l|}{ Night } \\
\hline mean & $118(3.3)$ & $119(6.1)$ & $117(3.8)$ & $123(2.6)$ & 0.22 & 0.71 \\
\hline $\max$ & $134(3.9)$ & $135(7.2)$ & $133(4.5)$ & $144(3.6)$ & 0.08 & 0.76 \\
\hline $\min$ & $102(3.1)$ & $104(5.5)$ & $100(3.9)$ & $105(2.2)$ & 0.35 & 0.61 \\
\hline \multicolumn{7}{|l|}{ Diastolic: } \\
\hline \multicolumn{7}{|l|}{ Day } \\
\hline mean & $79(2.1)$ & $82(3.7)$ & $77(2.5)$ & $76(2.2)$ & 0.25 & 0.32 \\
\hline $\max$ & $116(3.5)$ & $124(5.5)$ & $111(4.1)$ & $103(3.3)$ & 0.01 & 0.07 \\
\hline $\min$ & $60(1.9)$ & $62(3.8)$ & $59(1.9)$ & $59(2.5)$ & 0.67 & 0.33 \\
\hline \multicolumn{7}{|l|}{ Night } \\
\hline mean & $66(2.3)$ & $67(5.4)$ & $65(1.9)$ & $68(1.8)$ & 0.53 & 0.69 \\
\hline $\max$ & $80(3.4)$ & $78(6.2)$ & $82(4.1)$ & $88(4.1)$ & 0.14 & 0.58 \\
\hline $\min$ & $54(1.7)$ & $55(3.8)$ & $53(1.5)$ & $55(2.0)$ & 0.47 & 0.53 \\
\hline \multicolumn{7}{|c|}{ Mean blood pressure: } \\
\hline Day & $96(2.2)$ & 99 (4.3) & $95(2.4)$ & $98(3.3)$ & 0.67 & 0.35 \\
\hline Night & $84(2.4)$ & $86(5.2)$ & $83(2.3)$ & $88(1.6)$ & 0.21 & 0.58 \\
\hline \multicolumn{7}{|l|}{ Heart rate: } \\
\hline Day & $73(2.0)$ & $71(3.2)$ & $74(2.6)$ & $71(2.1)$ & 0.57 & 0.49 \\
\hline Night & $62(1.9)$ & $58(2.1)$ & $64(2.8)$ & $61(1.9)$ & 0.93 & 0.18 \\
\hline
\end{tabular}


one group than in the other. Like the authors of recent major studies in this field ${ }^{11}{ }^{13}{ }^{14}$ we therefore decided not to exclude these patients.

In fact, we found that in our study not only the number of treated patients, but also their drugs and dosage were almost equal in both groups. Moreover, the difference between the nocturnal dips in both groups remained significant whether these patients were included or not. We therefore believe that the influence of hypotensive treatment on the results may be neglected in our study.

Patients with NTG tend to have slightly lower diurnal and nocturnal blood pressure values than normal controls. In this study, however, statistically significant differences could only be found with maximal diastolic pressure values during the day, which, as an exception to the general trend, were even higher in the NTG group. Kaiser et al, ${ }^{13}$ who also continuously monitored blood pressure for 24 hours, reported significantly lower systolic blood pressure in NTG patients than in normals, but differences between diastolic values were not significant. We could not confirm this pronounced difference, though we found a weak similar trend. The disagreement between the study of Kaiser et al and ours may be related to a different composition of the patient groups rather than to a difference in study design or in patient number (which is smaller in our study). There may have been more non-FIG patients in Kaiser et als NTG group, who tend to have lower blood pressure than FIG patients according to our data. Additionally, there may be some differences in the selection of patients. Our NTG group comprised all patients (except two diabetics) hospitalised in 1994 because of a progressive visual field loss despite normal pressure, whereas we do not know whether Kaiser et al's NTG group was selected differently.

Graham et al also showed ${ }^{14}$ that mean blood pressure over 24 hours in 34 NTG patients was within normal limits for healthy people.

Focal ischaemic glaucoma, described first by Spaeth $^{16}$ is a typical vascular form of glaucoma with localised nerve fibre defects and almost normal IOP. Béchetoille and Bresson-Dumont ${ }^{11}$ reported lower blood pressure in patients with FIG compared with patients with high tension glaucoma. A comparison between FIG and nonFIG patients within the NTG group has not yet been made. We did not find significant differences between both groups, though there was a weak trend towards higher values in the FIG group. Patient numbers in both groups, however, are rather small. In summary, we did not find significantly lower blood pressure values in NTG patients than in normal controls.
Our study confirms, however, another observation, indicating greater blood pressure drops during the night in patients with normal tension glaucoma. ${ }^{14}$ These drops may, if autoregulation is abnormal, cause deterioration of optic nerve perfusion and thereby lead to nerve fibre damage. There is, however, large interindividual variability in blood pressure. Therefore, we believe that in the clinical examination of an individual patient moderately lower (nocturnal) blood pressure readings alone do not indicate a high risk for normal tension glaucoma.

1 Carter C, Brooks D, Doyle D, Drance S. Investigations into a vascular etiology for low-tension glaucoma. Ophthalmology 1990;97:49-55.

2 Hyong PFJ, de Jong N, Oosting H, Stilma J. Platelet aggregation, disc haemorrhage and progressive loss of visual gation, disc haemorrhage and progressive loss of
fields in glaucoma. Int Ophthalmol 1992;16:65-73.

3 Klaver JHJ, Greve EL, Goslinga H, Geijssen HC, Heuvelmans JHA. Blood and plasma viscosity measurements in patients with glaucoma. $\mathrm{Br} \mathcal{F}$ Ophthalmol 1985;69:765-70.

4 Cartwright MJ, Grajewski AL, Friedberg ML, Anderson DR, Richards DW. Immune-related disease and normaltension glaucoma. Arch Ophthalmol 1992;110:500-2.

5 Demailly P, Cambien F, Plouin PF, Baron P, Chevallier B. Do patients with low-tension glaucoma have particular Do patients with low-tension glaucoma have particular
cardiovascular characteristics? Ophthalmologica 1984;188: cardiovas

6 Corbett J, Phelps CD, Eslinger P, Montaguet PR. The neurologic evaluation of patients with low tension glaucoma. Invest Ophthalmol Vis Sci 1985;8:1101-4.

7 Gasser P, Flammer J. Blood-cell velocity in the nailfold capillaries of patients with normal-tension or high-tension

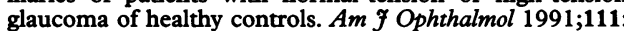
585-8.

8 Goldberg I, Hollows FC, Kass MA, Becker B. Systemic factors in patients with low-tension glaucoma. Br f Ophthalmol tors in patients with

9 Leighton DA, Phillips CI. Systemic blood pressure in openangle glaucoma, low tension glaucoma, and the normal eye. Brf Ophthalmol 1972;56:447-53.

10 Peräsalo R, Raitta C. Low blood pressure-a risk factor for nerve fiber loss in institutionalized geriatric glaucoma patients. Acta Ophthalmol (Suppl) 1990;68:65-7.

11 Béchetoille A, Bresson-Dumont H. Diurnal and nocturnal blood pressure drops in patients with focal ischemic glaucoma. Graefes Arch Clin Exp Ophthalmol 1994;232: 675-9.

12 Hayreh SS, Zimmermann MB, Podhajsky P, Alward WLM. Nocturnal arterial hypotension and its role in optic nerve head and ocular ischemic disorders. Am $\mathcal{f}$ Ophthalmol 1994;117:603-24.

13 Kaiser HJ, Flammer J, Graf T, Stümpfig D. Systemic blood pressure in glaucoma patients. Graefes Arch Clin Exp Ophthalmol 1993;231:677-80.

14 Graham SL, Drance SM, Wijsman K, Douglas GR, Mikelberg FS. Ambulatory blood pressure monitoring in glaucoma. The nocturnal dip. Ophthalmology 1995;102: in glauco

15 O'Brien E, Murphy J, Tyndall A. Twenty-four-hour ambulatory blood pressure in men and women aged 17 to 80 years: the Allied Irish Bank Study. $\mathcal{f}$ Hyperten 1991;9:355-60.

16 Spaeth GL. The pathogenesis of nerve damage in glaucoma: contribution of fluorescein angiography. New York: Grune \& Stratton, 1977: 89-91.

17 Spaeth GL. A new classification of glaucoma including focal glaucoma. Surv Ophthalmol (Suppl) 1994;38:9-17.

18 Richards AM, Nicholls MG, Espinger EA, Ikram H, Cullens $M$, Hinton D. Diurnal patterns of blood pressure, heart rate, and vasoactive hormones in normal man. Clin Exp Theory Pract 1986; 2:153-66.

19 Kaiser HJ, Flammer J. Systemic hypotension: a risk factor for glaucomatous damage. Ophthalmologica 1991;203:1058.

20 Tochikubo O, Minamisawa K, Miyakawa T. Blood pressure during sleep: antihypertensive medication. Am $\mathcal{f}$ Cardiol 1991;67:18-25. 